\title{
Inhibition of autophagy potentiates anticancer property of 20(S)-ginsenoside Rh2 by promoting mitochondria-dependent apoptosis in human acute lymphoblastic leukaemia cells
}

\author{
Ting Xia ${ }^{1, *}$, Jiancheng Wang, ${ }^{2,}$, Yingnan Wang ${ }^{2}$, Yuanyuan Wang ${ }^{2}$, Jianye Cai², \\ Min Wang ${ }^{1}$, Qidan Chen ${ }^{5}$, Jia Song ${ }^{1}$, Ziqi Yu ${ }^{1}$, Wei Huang ${ }^{1}$, Jianpei Fang ${ }^{3,4}$ \\ ${ }^{1}$ Key Laboratory of Industrial Fermentation Microbiology, Ministry of Education, College of Biotechnology, Tianjin University \\ of Science and Technology, Tianjin, P.R. China \\ ${ }^{2}$ Center for Stem Cell Biology and Tissue Engineering, Key Laboratory for Stem Cells and Tissue Engineering, Ministry of \\ Education, Sun Yat-Sen University, Guangzhou, P.R. China \\ ${ }^{3}$ Department of Pediatrics, Sun Yat-sen Memorial Hospital, Sun Yat-Sen University, Guangzhou, P.R. China \\ ${ }^{4}$ Key Laboratory of Malignant Tumor Gene Regulation and Target Therapy of Guangdong Higher Education Institutes, Sun \\ Yat-Sen University, Guangzhou, P.R. China \\ ${ }^{5}$ Department of Chemistry and Pharmacy, Zhuhai College, Jilin University, Zhuhai, P.R. China \\ *These authors have contributed equally to this work \\ Correspondence to: Jianpei Fang, e-mail: jpfang2005@163.com \\ Ting Xia, e-mail: xiatingsyu@foxmail.com
}

Keywords: ginsenoside Rh2, acute lymphoblastic leukaemia, autophagy, apoptosis, mitochondria

Received: September 10,2015 Accepted:March 01, $2016 \quad$ Published: March 23, 2016

\section{ABSTRACT}

Acute lymphoblastic leukaemia (ALL) is the most prevalent childhood malignancy. Although most children with ALL are cured, there is still a group of patients for which therapy fails owing to severe toxicities and drug resistance. Ginsenoside Rh2 (GRh2), a major bioactive component isolated from Panax ginseng, has been shown to have a therapeutic effect on some tumors. However, the molecular mechanisms of cell death induced by 20 (S)-GRh2 in ALL cells remains unclear. In this study, we showed that 20(S)-GRh2 inhibited the cell growth and induced mitochondria-dependent apoptosis and autophagy. But it has no cytotoxic effect on human normal blood cells. Furthermore, autophagy plays a protective role in 20(S)-GRh2-induced apoptosis in ALL cell lines and human primary ALL cells. We demonstrated that either genetic or pharmacologic inhibition of autophagy could be more effective in reducing viability and enhancing 20(S)-GRh2-induced toxicity than 20(S)-GRh2 treatment alone. In addition, inhibition of autophagy could aggravate mitochondrial ROS generation and mitochondrial damage, and then accelerate mitochondria-dependent apoptosis. Taken together, these results suggest that inhibition of autophagy can sensitize ALL cells towards 20 (S)-GRh2. The appropriate inhibition of autophagy could provide a powerful strategy to increase the potency of $20(\mathrm{~S})-\mathrm{GRh} 2$ as a novel anticancer agent for ALL therapy.

\section{INTRODUCTION}

Acute lymphoblastic leukaemia (ALL) is the most common malignancy in childhood, accounting for $25 \%$ of all childhood cancers [1]. It can be regarded as clonal expansion of B- and T-lymphocytes arrested at an immature stage of differentiation a malignant disorder of lymphoid progenitor cells [2]. Precursor B-acute lymphoblastic leukaemia (pre $\mathrm{B}-\mathrm{ALL}$ ) is the most prevalent phenotype of ALL, and T-acute lymphoblastic leukaemia (T-ALL) associated with a poor outcome [3, 4]. However, current standard treatment consists of combination chemotherapy that is highly toxic to growing children, both in short and long terms [5-7]. To avoid serious side effects in chemotherapy and improve survival rates, novel anticancer agents are needed to address the emerging problem. Recent studies have shown that several natural traditional Chinese medicine (TCM) products can activate cell death pathway in cancer cells $[8,9]$. 
Ginsenosides are extracted from the root of Panax ginseng C.A. Meyer, which are the major effective ingredients in ginseng $[10,11]$. Based on the structural differences, ginsenosides can be classified 20(S)protopanaxadiol (ginsenosides Rb1, Rb2, Rb3, Rc, Rd, Rh2, Rg3 and others) or 20(S)-protopanaxatriol (ginsenosides Re, Rg1, Rg2, Rh1 and others) compounds [12, 13]. Among these, GRh2 has been shown to have anticancer effects, and could inhibit cell growth and induce apoptosis in several cancer cell lines [13-16]. Furthermore, it has been reported that 20(S)-GRh2 exhibits stronger anticancer activity than 20(R)-GRh2 [17-18]. Due to its safety and no side-effects, GRh2 could be further developed as a useful drug for cancer therapy $[19,20]$. Recently, researchers have found that 20(S)-GRh2 could induce autophagy when undergoing induced apoptosis in Hepatocellular carcinoma [21]. Our previous studies demonstrated that 20(S)-GRh2 could inhibit cell growth and induce apoptosis in pre B-ALL Reh cells [22]. In this report, we are interested to investigate whether autophagy was intervened in 20(S)-GRh2-induced apoptosis in human ALL cells.

Autophagy and apoptosis are catabolic pathways essential for organismal homeostasis, both of which play key roles in ultimate decisions of cancer cell fate [23]. Apoptosis, or type I PCD, involves the degradation of cellular constituents by a family of cysteine proteases called caspases [24]. As we all known, two core pathways exist to induce apoptosis, the extrinsic-death receptor pathway and intrinsic-mitochondrial pathway [25]. The mitochondrial pathway of apoptosis is seen as a common cause of tumor cell death induced by chemotherapeutic agents [26, 27]. The mitochondrial apoptotic pathway is characterized by reactive oxygen species (ROS) accumulation, loss of mitochondrial membrane potential (MMP), release of cytochrome c into the cytoplasm, which is followed by activation of caspase- 9 and caspase-3 [28-30].

Similarly, autophagy is an evolutionarily conserved catabolic process of lysosomal degradation of cytoplasmic content in eukaryotes [31]. It is regulated by proteins including mammalian target of rapamycin (mTOR), phosphoinosmde-3-kinase (PI3K), GTPases, calcium and elements of protein synthesis machinery [32]. Recent studies have demonstrated that the existence of a complex relationship between autophagy and apoptosis, determining whether a cell will live or die in response to anticancer therapies [33, 34]. On one hand, autophagy can clear toxic elements and damage organelles, which suppresses apoptosis and prevents cell death [35]. On the other hand, it could also act as a facilitator of apoptosis via damaging mitochondria and contribute to cell death [36]. However, the molecular mechanisms of the interaction between autophagy and apoptosis induced by $20(\mathrm{~S})-\mathrm{GRh} 2$ remain unclear.

In the present study, we used autophagy inhibitor 3-methyladenine (3-MA), autophagy stimulator rapamycin (RAPA) and ATG5 knockdown to evaluate the potential role of autophagy in 20(S)-GRh2-induced apoptosis. We examined mitochondrial function and ROS generation to elucidate the interaction between autophagy and apoptosis induced by $20(\mathrm{~S})-\mathrm{GRh} 2$ in ALL. These findings would illuminate the anticancer property of $20(\mathrm{~S})-\mathrm{GRh} 2$ and provide a promising strategy for treatment of ALL.

\section{RESULTS}

\section{Effect of 20(S)-GRh2 on cell proliferation in ALL cell lines and human normal blood cells}

The effect of 20(S)-GRh2 on cell viability in ALL cell lines and human normal blood cells were investigated by cell counting kit-8 (CCK-8) assay. Cells were treated with increasing concentrations of 20(S)-GRh2 for $24 \mathrm{~h}$. The results showed that 20(S)-GRh2 significantly reduced the viability of ALL cell lines in a dose-dependent manner (Figure 1A \& 1B). The IC50 of 20(S)-GRh2 was about $40 \mu \mathrm{M}$ for Reh cells and $35 \mu \mathrm{M}$ for Jurkat cells. However, the viability of normal Band T- cells were not obviously decreased (Figure 1D \& 1E).

Then we chose $40 \mu \mathrm{M}$ GRh2 to detect the cell growth at different time points. When cells were treated with $40 \mu \mathrm{M} 20$ (S)-GRh2 for $48 \mathrm{~h}$, the viability of Reh and Jurkat cells were decreased in a time-dependent manner (Figure 1C), whereas in normal B- and T- cells, the cell viability were not significantly decreased. (Figure 1F). Collectively, these results suggest that 20(S)-GRh2 inhibits cell proliferation of ALL cell lines and has no cytotoxic effect on human normal blood cells.

\section{0(S)-GRh2 induces mitochondria-dependent apoptosis in ALL cells}

First, we examined apoptotic cells induced by 20(S)GRh2 via Annexin V and 7-AAD double staining. Cells were treated with $40 \mu \mathrm{M} 20(\mathrm{~S})-\mathrm{GRh} 2$ for $48 \mathrm{~h}$, and Annexin $\mathrm{V}$ positive cells were measured with flow cytometric analysis. The results showed that in Reh and Jurkat cells, the population of Annexin V positive cells was $16.66 \pm$ $3.25 \%$ and $20.70 \pm 3.22 \%$ at $24 \mathrm{~h}$, respectively. After a $48 \mathrm{~h}$ treatment with $40 \mu \mathrm{M} 20(\mathrm{~S})$-GRh2, the percentage of apoptotic cells was further increased to $50.87 \pm 5.43 \%$ at $24 \mathrm{~h}$ and $47.72 \pm 5.02 \%$, respectively. However, in normal B- and T- cells, the apoptotic levels were not significantly increased after $24 \mathrm{~h}$ and $48 \mathrm{~h}$ 20(S)-GRh2 administration (Figure 2A \& 2B). These data indicate that 20(S)-GRh2 can induce cell death in ALL cells but not in normal blood cells.

Then, in order to measure mitochondrial ROS levels triggered by 20(S)-GRh2 in ALL cells, we used MitoSOX ${ }^{\mathrm{TM}}$ Red reagent, which can detect superoxide inside mitochondria of live cells. The results showed that the red fluorescence intensity was obviously increased in 20(S)GRh2-treated Reh and Jurkat cells, and then markedly attenuated when concurrent treatment with mitoTEMPO, a specific mitochondrial ROS inhibitor (Figure 2C). In addition, the levels of mitochondrial ROS were not obviously 
increased, when normal B- and T- cells were treated with $40 \mu \mathrm{M}$ 20(S)-GRh2 for $24 \mathrm{~h}$ (Supplementary Figure S2A \& S2B). On one hand, mitochondrial damage could lead to mitochondrial dysfunction. We used the sensitive fluorescent probe JC-1 as an indicator of mitochondrial dysfunction to examine the MMP levels. In fluorescence microscopic study, 20(S)-GRh2 induced a shift in the fluorescence spectrum of JC-1 from an aggregated form with orange-red fluorescence to a monomeric form with green fluorescence (Figure 2D \& 2E), while mitoTEMPO rescued the reduction of red/green ratio in $20(\mathrm{~S})-\mathrm{GRh} 2$ treated Reh and Jurkat cells (Figure 2F). On the other hand, mitochondrial damage could trigger intrinsic apoptosis. We analyzed the expression of mitochondrial apoptosis-related proteins in 20(S)-GRh2-treated cells by western blot. We found that the expression levels of cytochrome c, cleaved caspase- 9 and -3 proteins were upregulated; whereas the expression levels of these proteins were decreased by adding mitoTEMPO in 20(S)-GRh2-treated Reh cells (Supplementary Figure S1A \& S1B). Therefore, these results suggest that $20(\mathrm{~S})-\mathrm{GRh} 2$ induces apoptosis through generation of mitochondrial ROS and mitochondrial signaling pathways in ALL cells.

\section{0(S)-GRh2 induces autophagy in ALL cells}

Firstly, we used monodansylcadaverine (MDC), a lysosomotropic compound to assess autophagy induction via the accumulation of MDC-labeled vacuoles. Reh cells were treated with increasing concentrations of 20(S)GRh2 and stained with MDC, a fluorescent dye that selectively labels autophagic vacuoles (AVOs). As shown in Supplementary Figure S3A, in laser scanning confocal microscope study, MDC staining revealed that huge amount of AVOs formation occurred in Reh cells after 24 h 20(S)-GRh2 treatment. Consistent with this finding, in flow cytometric study, the percentage of MDC positive cells was also increased after 20(S)-GRh2 treatment (Supplementary Figure S3B \& S3C).

To determine whether 20(S)-GRh2 treatment increased the autophagic flux in Reh and Jurkat cells, we conducted an analysis using autophagy protein LC3II by western blot. As shown in Reh and Jurkat cells, the expression levels of LC3-II were increased at $24 \mathrm{~h}$ after treatment with increasing concentrations of 20(S)-GRh2 (Figure 3A \& 3B). These data indicate that 20(S)-GRh2 treatment induces autophagic flux in ALL cells. Next, ultrastructural analysis by electron microscopy also revealed the formation of autophagosome in Reh and Jurkat cells at $24 \mathrm{~h}$ after 20(S)-GRh2 treatment (Figure 3C).

For further confirmation of autophagy, we performed an LC3 puncta assay by using pEGFP-LC3 and chloroquine (CQ), which inhibits late phase autophagy. The results showed that in Reh and Jurkat cells, 20(S)GRh2 treatment significantly increased the number of GFP-LC3 puncta per cell compared with control, and CQ further enhanced the GFP-LC3 positive puncta upon 20(S)-GRh2 treatment (Figure 3D \& 3E). Taken together,
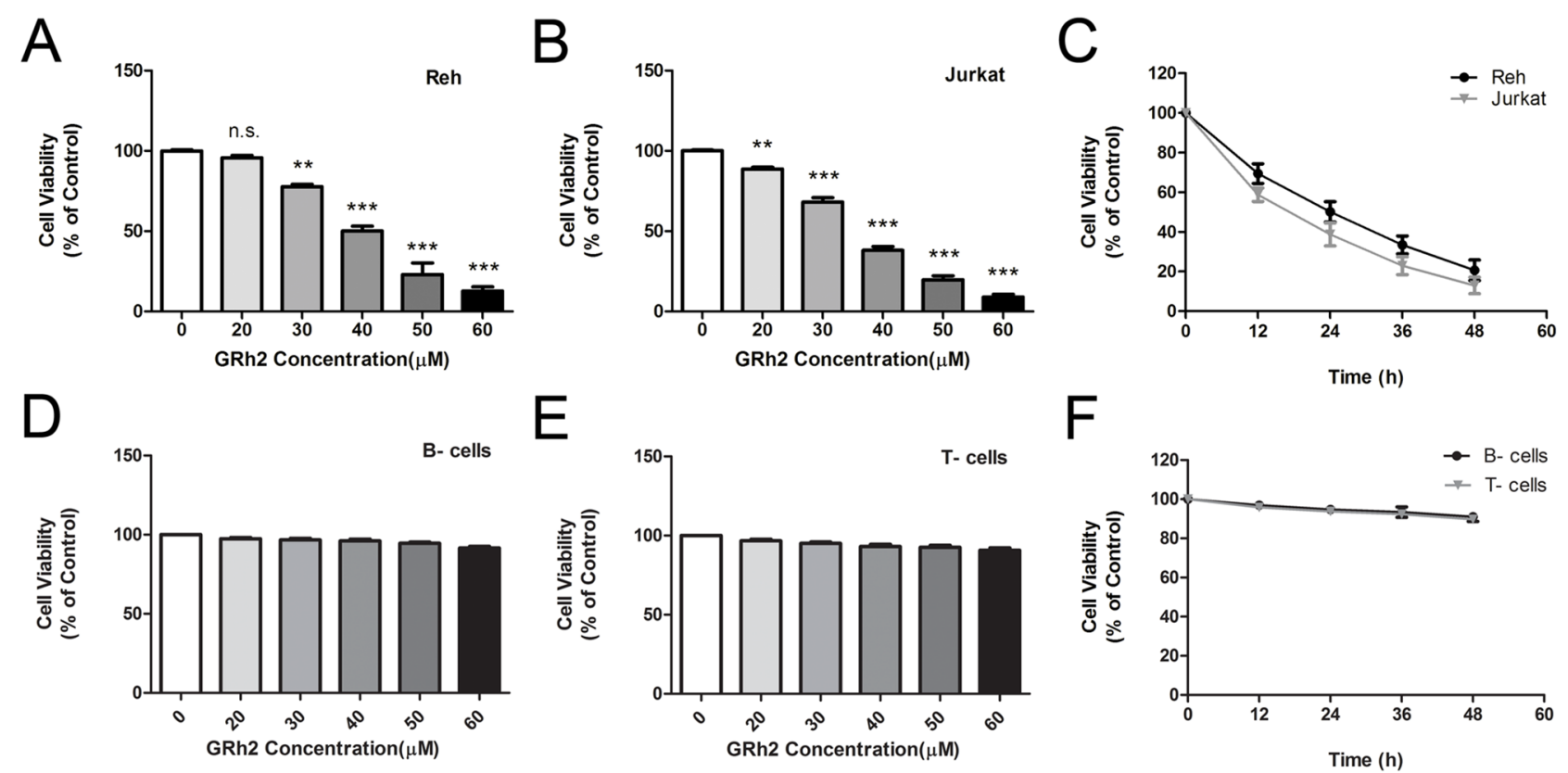

Figure 1: Effect of 20(S)-GRh2 on cell viability in ALL cell lines and human normal blood cells Reh. A. and Jurkat B. cells were treated with various concentrations of 20 (S)-GRh2 $(0,20,30,40,50$ and $60 \mu \mathrm{M})$ for $24 \mathrm{~h}$. C. Reh and Jurkat cells were treated with $40 \mu \mathrm{M} 20$ (S)-GRh2 for different times (12, 24, 36 and $48 \mathrm{~h}$ ). The cell viability was assessed with cell counting kit-8 assay. Human normal B- cells D. and T- cells E. were treated with various concentrations of 20(S)-GRh2 for $24 \mathrm{~h}$. F. Normal B- and T- cells were treated with 40 $\mu \mathrm{M} 20$ (S)-GRh2 for different times. Cell viability of every group was determined separately by cell counting kit- 8 assay. Data are presented as mean $\pm \operatorname{SEM}(\mathrm{n}=3)$ for each group. $* * \mathrm{p}<0.01, * * * \mathrm{p}<0.001$. 

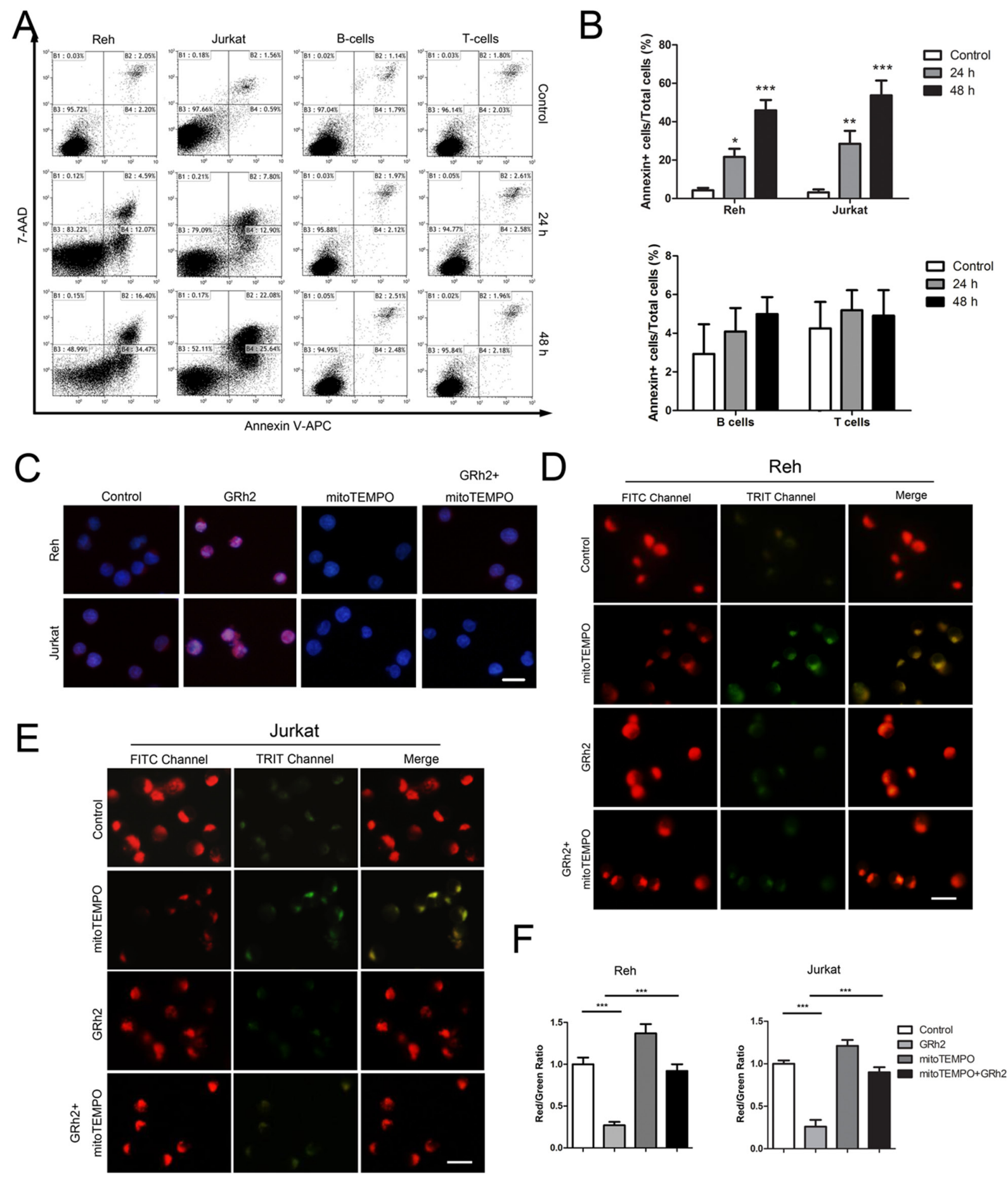

Figure 2: GRh2 induces mitochondria-dependent apoptosis in ALL cells. A. Cells were treated with $40 \mu \mathrm{M} 20(\mathrm{~S})-\mathrm{GRh} 2$ and harvested at the indicated time points. Representative dot plots of Annexin V/7-AAD staining are shown. Annexin V positive cells were analyzed by flow cytometer. B. The corresponding histograms were quantified by Image J. Data are reported as mean \pm SEM ( $n=3$ ) for each group. ${ }^{* *} \mathrm{p}<0.01, * * * \mathrm{p}<0.001$ : Bonferroni's post hoc test vs. control. C. Reh and Jurkat cells were treated with $40 \mu \mathrm{M} 20(\mathrm{~S})-\mathrm{GRh} 2$ for $24 \mathrm{~h}$ in the presence or absence of $50 \mu \mathrm{M}$ mitoTEMPO. Cells were stained with MitoSOX ${ }^{\mathrm{TM}}$ Red and Hoechst 33342 . The fluorescence intensity was evaluated using fluorescent microscopy $(\mathrm{Bar}=10 \mu \mathrm{M})$. Reh D. and Jurkat E. cells were treated with different ligands as previously described and stained with JC-1. The fluorescence intensity was evaluated using fluorescent microscopy (Bar=10 $\mu \mathrm{M})$. F. The ratio of Red/ Green reflects MMP levels. The corresponding histograms were quantified by Image J. All data are represented as mean \pm SEM ( $\mathrm{n}=3$ ) for each group. 
A

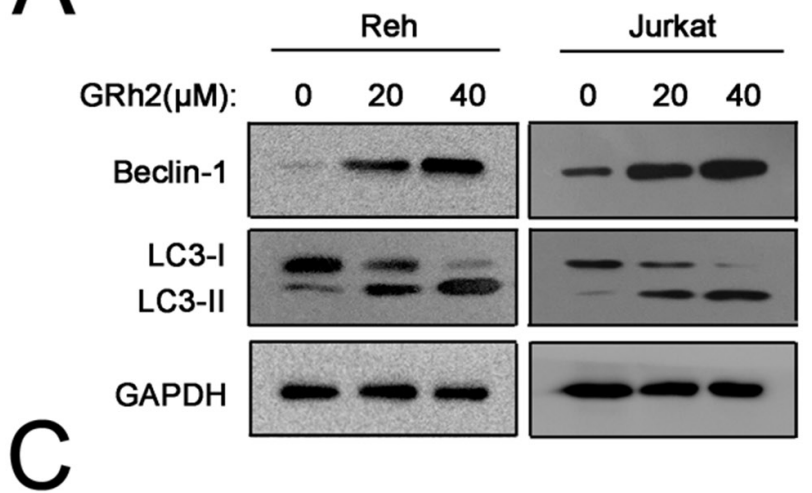

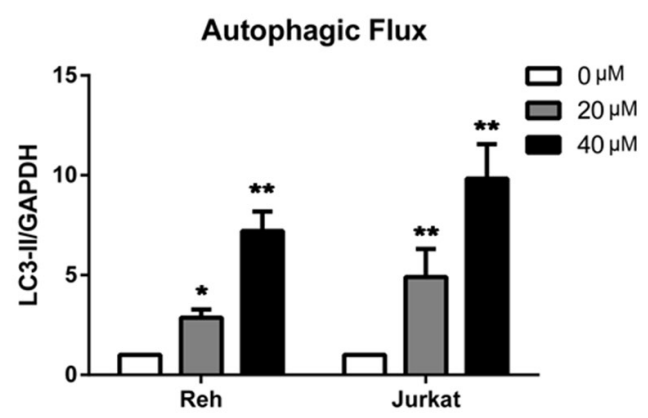

Reh
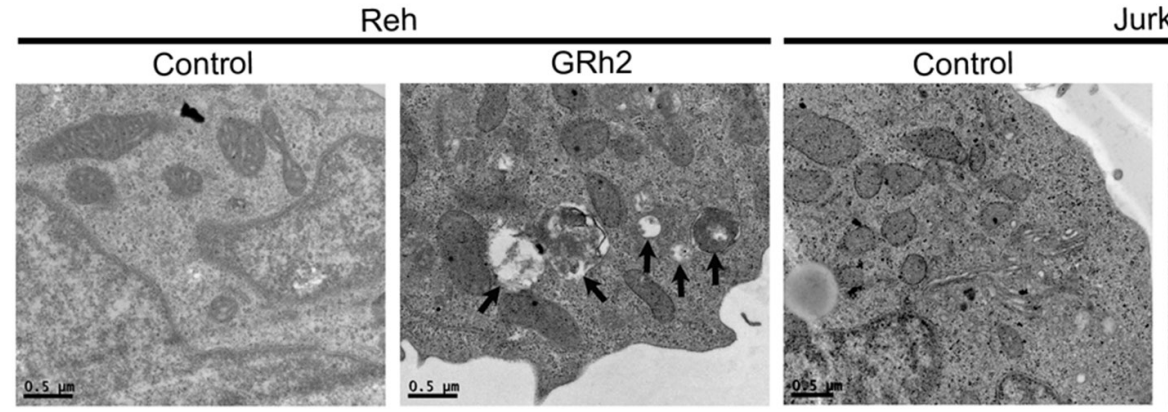

Jurkat
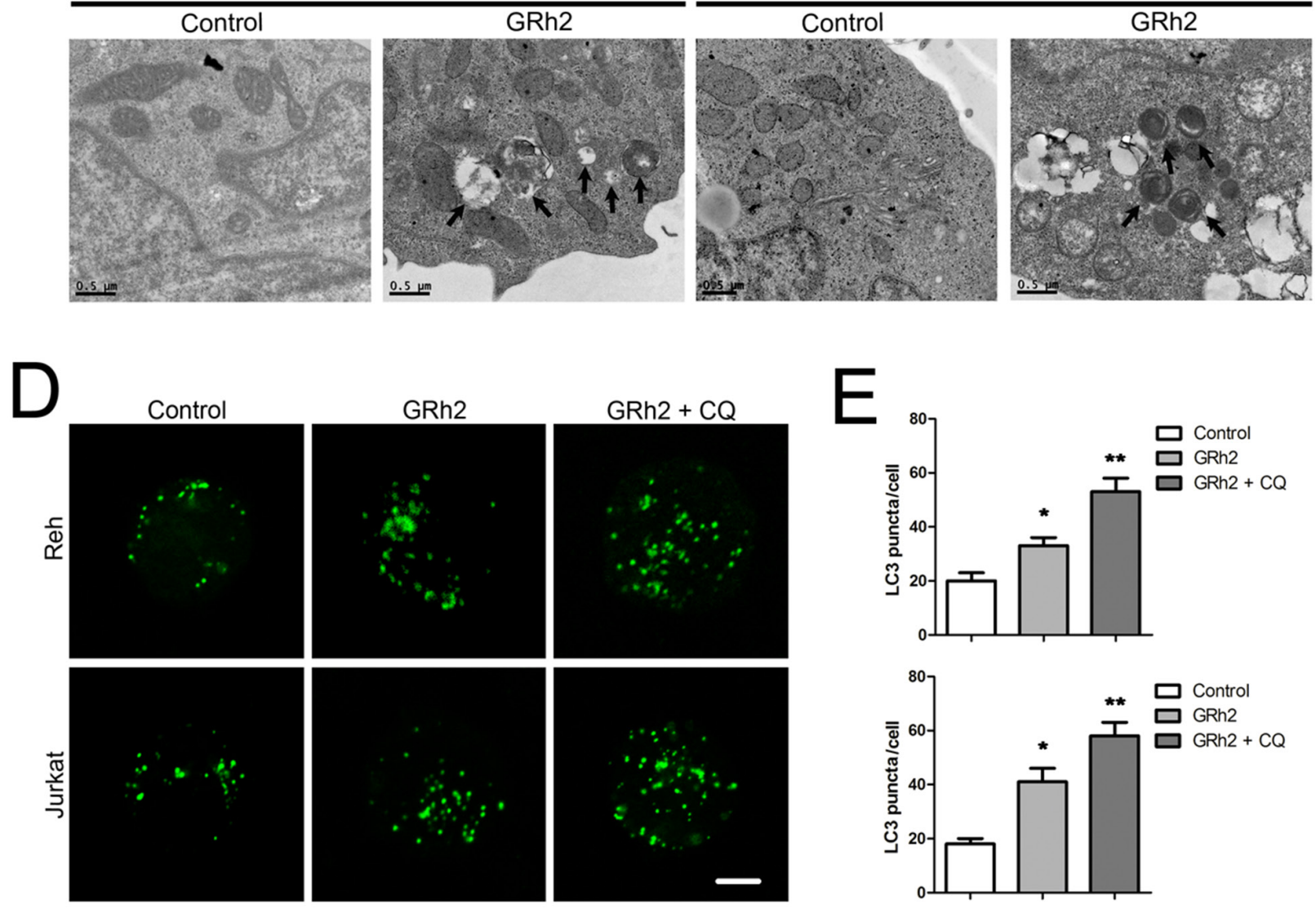

Figure 3: 20(S)-GRh2 induces autophagy in ALL cells. A. Reh and Jurkat cells were treated with different concentrations of 20(S)-GRh2 for 24h. The expression levels of LC3 were detected by western blot analysis. GAPDH was used as a loading control. B. Intensity of LC3-II and GAPDH levels was determined using densitometry analysis. Bar graphs shown represent normalized intensity levels of LC3-II (LC3-II/GAPDH) relative to no treatment control. Data are presented as mean \pm SEM $(n=3)$ for each group. $* \mathrm{p}<0.05, * * \mathrm{p}<0.01$. C. Changes of ultrastructure in control and $40 \mu \mathrm{M} 20(\mathrm{~S})$-GRh2 group were observed by transmission electron microscope $(\mathrm{Bar}=0.5 \mu \mathrm{M})$. Arrows indicate autophagosomes in 20(S)-GRh2-treated ALL cells. D. Cells were transfected with pEGFP-LC3 plasmids. After $24 \mathrm{~h}$ transfection, cells were treated with $40 \mu \mathrm{M} 20(\mathrm{~S})$-GRh2 for $24 \mathrm{~h}$ in the presence or absence of $50 \mu \mathrm{M} \mathrm{CQ}$. The fluorescence of GFP-LC3 was examined by laser scanning confocal microscope (Bar=5 $\mu \mathrm{M})$. E. GFP-LC3 puncta in each cell was determined and bar graphs are representative of 100 cells. Data are presented as mean \pm SEM $(n=3)$ for each group. $* \mathrm{p}<0.05, * * \mathrm{p}<0.01$. 
these results suggest that 20(S)-GRh2 can also induce autophagy in ALL cells.

\section{Autophagy is involved in 20(S)-GRh2-induced toxicity in ALL cells}

We used 3-MA and RAPA to investigate the role of autophagy in 20(S)-GRh2-induced toxicity. 3-MA, an autophagic inhibitor, interferes with the formation of autophagosomes in mammalian cells via inhibition of the class III PI3K activity [37]. RAPA, an autophagic inducer, could inhibit mTOR, thus enhancing autophagy [38]. The involvement of autophagy was confirmed using LC3 puncta assay. As shown in Reh and Jurkat cells, the number of GFPLC3 puncta per cell was significantly increased in cells treated with 3-MA and 20(S)-GRh2, while markedly decreased in cells treated with RAPA and 20(S)-GRh2, compared to 20(S)GRh2 alone (Supplementary Figure S4A \& S4B). In addition, the number of GFP-LC3 puncta per cell was not significantly increased after 24 h 20(S)-GRh2 administration, while markedly increased in normal B- and T-cells treated with RAPA compared with control (Supplementary Figure S2C \& S2D).

Then, combination of 3-MA and 20(S)-GRh2 was most effective in reducing cell viability compared with 20(S)-GRh2 alone, while induction of autophagy by RAPA significantly improved cell viability in the presence of $20(\mathrm{~S})-\mathrm{GRh} 2$ (Figure 4A). Moreover, combination of 3-MA and 20(S)GRh2 effectively increased the percentage of apoptotic cells compared with 20(S)-GRh2 treatment alone, while induction of autophagy by RAPA dramatically attenuated apoptotic cell death (Figure 4C \& 4D). These results indicate that inhibition of autophagy by 3-MA accelerates 20(S)-GRh2-induced toxicity to ALL cells, while induction of autophagy by RAPA protects ALL cells against 20(S)-GRh2-induced toxicity.

Next, to verify involvement of autophagy in 20(S)GRh2-induced cytotoxicity, we specifically inhibited autophagy with shRNA targeting essential autophagyrelated protein ATG5 in combination with 20(S)-GRh2. The results showed that shATG5 combined with 20(S)GRh2 treatment markedly reduced the viability of Reh and Jurkat cells compared with cells treated with 20(S)-GRh2 and vector (Figure 4B). Furthermore, we used the LIVE/ $D E A D \AA$ viability/cytotoxicity assay to quantitate cell death. The results showed that combined treatment of autophagy inhibition with shATG5 and 20(S)-GRh2 significantly increased the percentage of dead cells compared with 20(S)GRh2 and vector treatment (Figure 4E and Supplementary Figure S5A). These results suggest that in Reh and Jurkat cells, ATG5 knockdown can more effectively decrease cell viability and accelerate apoptotic cell death upon 20(S)GRh2 treatment. Taken together, autophagy acts as an adaptive way to protect ALL cells against 20(S)-GRh2induced cytotoxicity. Either genetic or pharmacologic inhibition of autophagy combined with 20(S)-GRh2 can be more effective in exacerbating 20(S)-GRh2-induced cytotoxicity than treatment with 20(S)-GRh2 alone.
Inhibition of autophagy accelerates 20(S)-GRh2induced apoptosis via mitochondrial ROS and mitochondrial damage

To investigate the mechanisms of 20(S)-GRh2induced toxicity to ALL cells, we measured mitochondrial ROS levels, MMP and mitochondrial-related apoptosis proteins. We found that in Reh cells, mitochondrial ROS generation was markedly increased in cells treated with 3-MA and 20(S)-GRh2, but significantly decreased in cells treated with RAPA and 20(S)-GRh2, compared to 20(S)GRh2 alone (Figure 5A \& 5B). Furthermore, combined treatment of 3-MA and 20(S)-GRh2 markedly accelerated depolarization of MMP, but combination of RAPA and 20(S)-GRh2 significantly attenuated it, compared with 20(S)-GRh2 treatment alone (Figure 5C \& 5D). In addition, combined treatment of 3-MA and 20(S)-GRh2 dramatically increased the expression levels of mitochondria-associated pro-apoptotic proteins compared with 20(S)-GRh2 treatment alone, while combination of RAPA and 20(S)GRh2 markedly decreased the expression levels of these proteins in Reh cells (Supplementary Figure S6A \& S6B).

Next, we used shRNA to knockdown autophagy gene products ATG5 to confirm the role of mitochondrial ROS in 20(S)-GRh2-induced toxicity. The results showed that in Reh cells, shATG5 combined with 20(S)-GRh2 treatment markedly increased the fluorescence intensity compared with cells treated with 20(S)-GRh2 and control vector (Supplementary Figure S7A \& S7B). In addition, combined treatment of autophagy inhibition with shATG5 and 20(S)-GRh2 significantly accelerated the loss of MMP compared with 20(S)-GRh2 and control vector treatment (Supplementary Figure S7C \& S7D). These results taken together indicate that either genetic or pharmacologic inhibition of autophagy combined with 20(S)-GRh2 aggravates accumulation of mitochondrial ROS and mitochondrial damage, thus promoting apoptosis.

\section{Effect of autophagy on 20(S)-GRh2-induced apoptosis in primary ALL cells}

We also evaluated the effect of autophagy on 20(S)GRh2-induced apoptosis in primary ALL cells. Firstly, we investigated whether treatment with $20(\mathrm{~S})$-GRh2 could induce autophagy in primary ALL cells. As shown in Figure $6 \mathrm{~A} \& 6 \mathrm{~B}$, both primary B- ALL and T- ALL cells treated with 20(S)-GRh2 showed increased number of GFP-LC3 puncta per cell compared with untreated cells. CQ further enhanced GFP-positive puncta upon 20(S)GRh2 treatment, indicating that 20(S)-GRh2 treatment induced autophagic flux. Next, we used CQ to determine the effect of autophagy on 20(S)-GRh2-induced apoptosis in primary ALL cells. Our data showed that 20(S)-GRh2 treatments alone significantly increased the percentage of apoptotic cells compared with control, while the combination of CQ and 20(S)-GRh2 further increased the 
apoptotic levels (Figure 6C \& 6D). These results taken together indicate that inhibition of autophagy by $\mathrm{CQ}$ exacerbates 20(S)-GRh2-induced apoptosis in primary ALL cells.

\section{DISCUSSION}

ALL is the most common childhood tumor, accounting for almost $30 \%$ of pediatric cancers [39]. As a step in overcoming the limitation in chemotherapy, innovative approaches are needed to further improve survival rates and to avoid serious side effects. Ginsenosides, the major pharmacologically active ingredients of ginseng, have been shown to have multiple medical effects such as anti-oxidative, anti-inflammation, anticancer and other health-improving activities [40, 41]. GRh2 is a pure compound extracted from ginsenosides and has anticancer activity [13-16, 42]. In this study, our objective is to explore the relationship between apoptosis and autophagy in 20(S)-GRh2-treated ALL cells, which could provide a novel anticancer agent for ALL therapies.
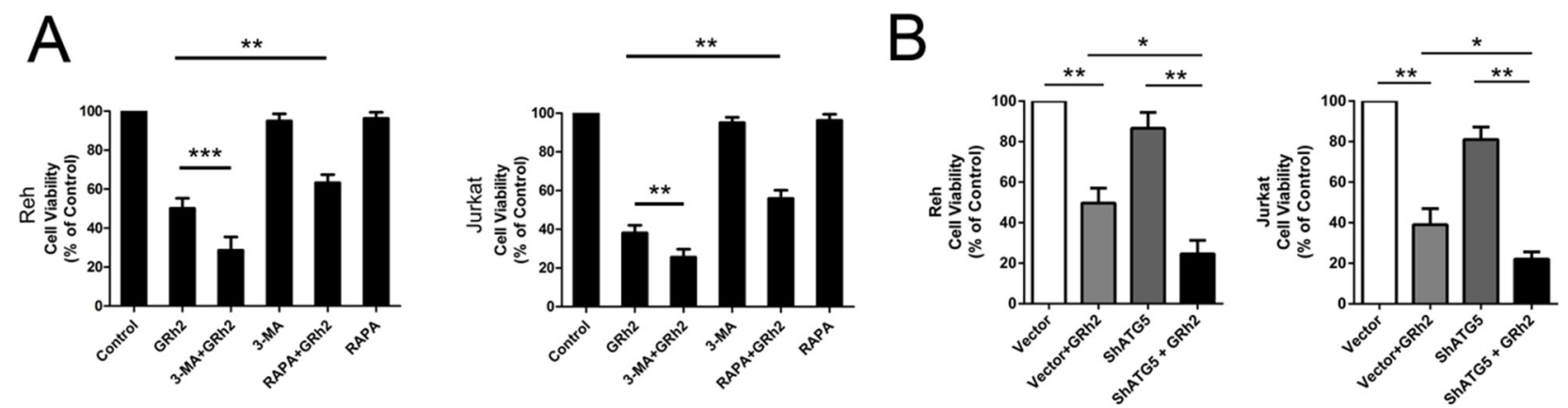

C
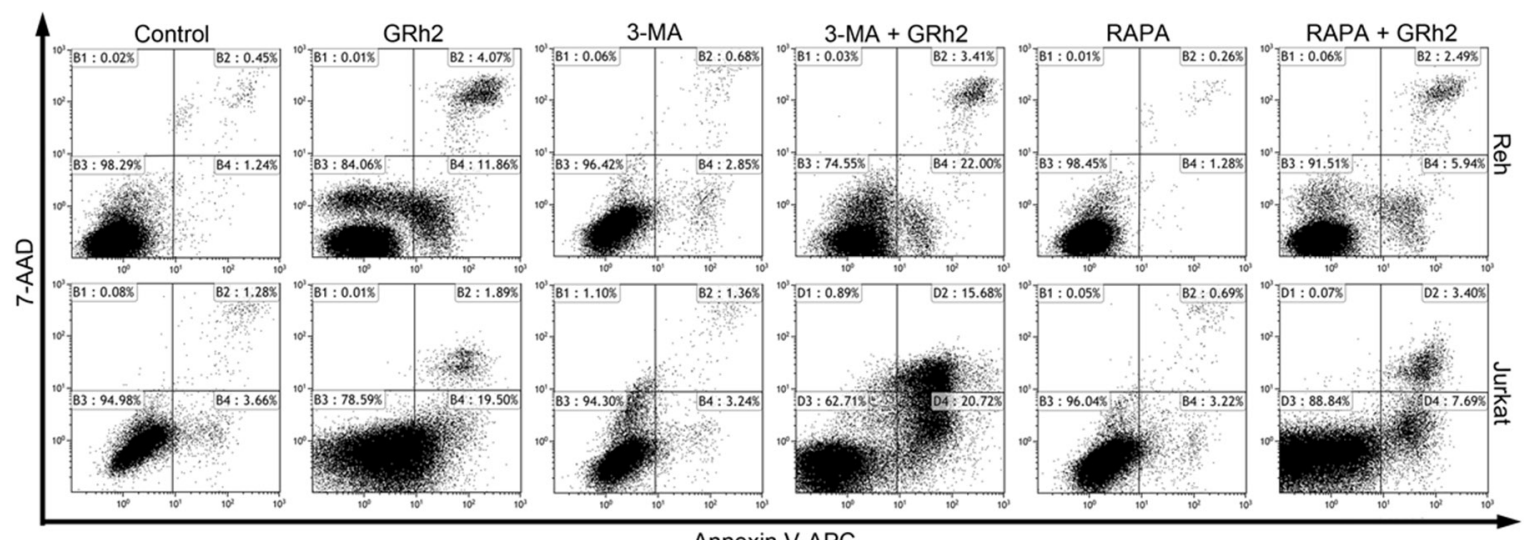

Annexin V-APC
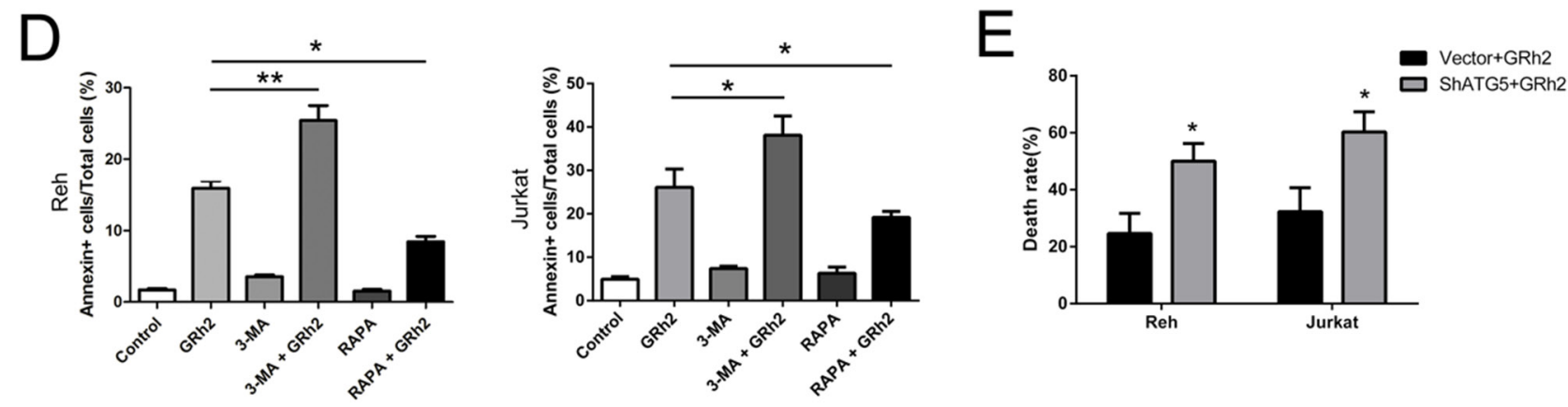

Figure 4: Autophagy is involved in 20(S)-GRh2-induced toxicity in ALL cells. Cell viability was determined in Reh and Jurkat cells by the CCK-8 assay. A. Cells were treated with $40 \mu \mathrm{M} 20(\mathrm{~S})-\mathrm{GRh} 2$ in the presence or absence of 3-MA or RAPA for $24 \mathrm{~h}$. B. Cells transfected with vector or ShATG5 plasmid were exposed to $40 \mu \mathrm{M} 20(\mathrm{~S})-\mathrm{GRh} 2$ for $24 \mathrm{~h}$. Data are presented as mean $\pm \mathrm{SEM}$ ( $\mathrm{n}=3$ ) for each group. $* \mathrm{p}<0.05, * * \mathrm{p}<0.01, * * * \mathrm{p}<0.001$. C. The percentage of apoptotic cells was determined by FACS analysis. The flow cytometry dot plots showed the analysis of the percentage of apoptotic cells within ALL cell populations. D. The percentage of apoptotic cells in every group was analyzed and graphed. Data are presented as mean $\pm \operatorname{SEM}(\mathrm{n}=3)$ for each group. $* \mathrm{p}<0.05,{ }^{*} \mathrm{p}<0.01$. E. Cells transfected with vector or ShATG5 plasmid were exposed to $40 \mu \mathrm{M} 20$ (S)-GRh2 for $24 \mathrm{~h}$. The percentage of dead cells with EthD-1 probe was determined by fluorescent microscopy. Images were analyzed using Image J. A minimum 500 cells were counted. 
Recently, it has been reported widely that GRh2 could inhibit tumor cells proliferation and induce tumor cells apoptosis [43-45]. The results presented here suggested that in human ALL cells, 20(S)-GRh2 inhibited cell growth in a dose- and time-dependent manner, while had no cytotoxic ability to human normal blood cells. Both apoptosis and autophagy play essential roles in the development of organs, homeostasis, and cancer. A comprehensive understanding of autophagy and apoptosis is essential for the development of effective cancer therapeutics [46, 47]. Consistent with previous studies, our data were confirmed that 20(S)-GRh2 induced apoptosis through mitochondrial signaling pathway in ALL cells. Meanwhile, we reported a novel function of 20(S)-GRh2, induction of autophagy, as shown by cell morphological changes and increased autophagic flux in ALL cells. But 20(S)-GRh2 can not induce autophagy in normal blood cells. Therefore, we demonstrated that autophagy and apoptosis were both induced by 20(S)-GRh2 in ALL cells.

The intricate relationship between autophagy and apoptosis is complex and varies with cell and stress distinction [48, 49]. Some recent studies have focused on the intricate relationship between drug-induced autophagy and apoptosis. Several contradictory views of the effects of autophagy on chemotherapy-induced apoptosis in cancer have been reported [50, 51]. Chemotherapy-induced autophagy protects cells from apoptosis in some contexts [52], but promotes apoptosis in the others [53]. To explore the interplay between apoptosis and autophagy in 20(S)GRh2-treated ALL cells, we used 3-MA, RAPA and ATG5
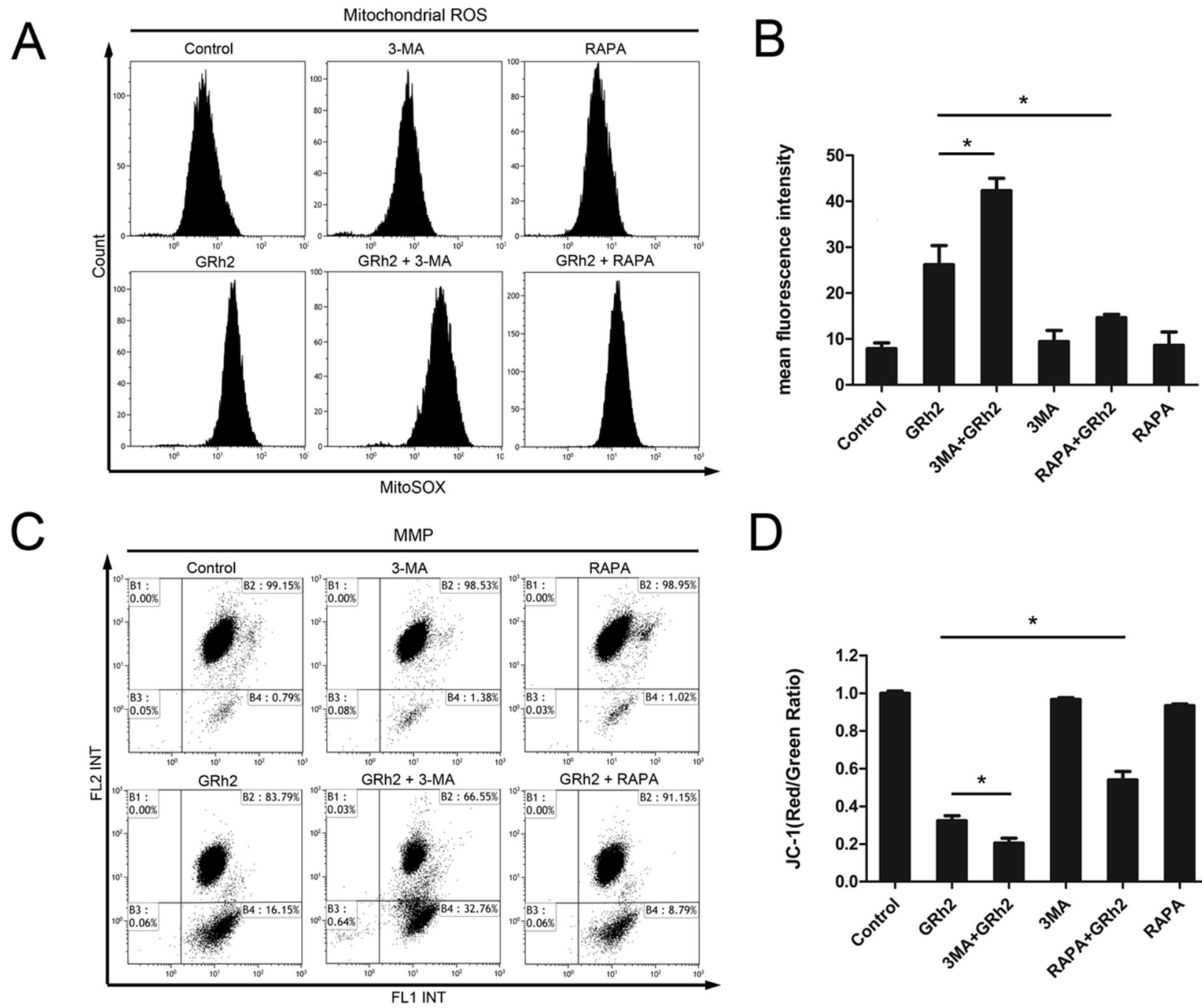

D

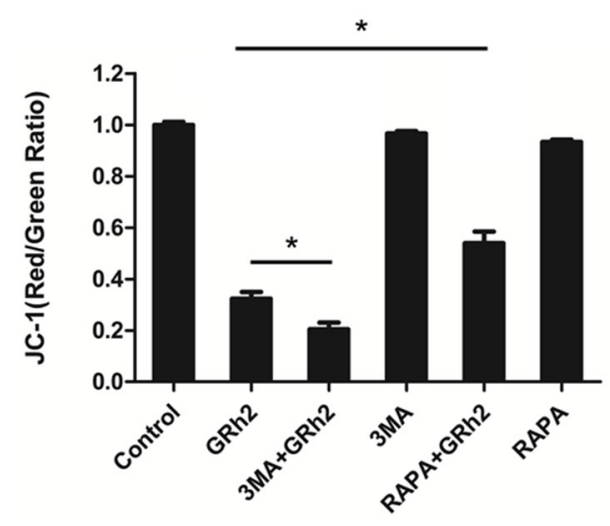

Figure 5: Inhibition of autophagy accelerates 20(S)-GRh2-induced apoptosis via mitochondrial ROS and mitochondrial damage. Reh cells were treated with $40 \mu \mathrm{M}$ 20(S)-GRh2 in the presence or absence of 3-MA or RAPA for 24 h. A. The MitoSOX ${ }^{\mathrm{TM}}$ Red fluorescence intensity was detected by flow cytometry. B. The corresponding histograms were quantified by Image J. All data are represented as mean $\pm \operatorname{SEM}(n=3)$ for each group. ${ }^{*} \mathrm{p}<0.05$. C. Representative pictures of JC-1 staining were shown, and the fluorescence intensity was detected by flow cytometry. D. The ratio of FL2/FL1 was reflects MMP levels. Data are represented as mean $\pm \mathrm{SEM}$ ( $\mathrm{n}=3$ ) for each group. ${ }^{*} \mathrm{p}<0.05$. 
knockdown to manipulate autophagy. We demonstrated that inhibition of autophagy by 3-MA sensitizes ALL cells to 20(S)-GRh2, while induction of autophagy by RAPA protects cells against apoptosis. In addition, we found that ATG5 knockdown further enhanced cytotoxicity of 20(S)-GRh2 to ALL cells. Therefore, these findings suggest that autophagy plays a protective role in 20(S)-GRh2-induced apoptosis, and either genetic or pharmacologic inhibition of autophagy can effectively sensitize ALL cells to 20(S)-GRh2.

Then, to illuminate the molecular mechanisms of interaction between apoptosis and autophagy in 20(S)-GRh2-treated ALL cells, we further investigated mitochondrial ROS levels and mitochondrial dysfunction. ROS mainly generate inside mitochondria, whereas excess ROS generation could cause mitochondrial damage $[54,55]$. Some links between apoptosis and autophagy are indicated via mitochondria [56]. It is reported that the elimination of damaged mitochondria by autophagy would prevent the release of proapoptotic substances from mitochondria, thus preventing apoptosis. In the absence of such clean up, the release of molecules like cytochrome $\mathrm{c}$ and apoptosis-inducing factor (AIF) from damaged mitochondria would lead to apoptosis $[57,58]$. In this study, we found that inhibition of autophagy combined with 20(S)-GRh2 markedly increased mitochondrial ROS generation and accelerated dissipation of MMP in ALL cells, while induction of autophagy combined with 20(S)-GRh2 significantly alleviated 20(S)-GRh2-induced mitochondrial ROS generation and mitochondrial damage. In addition, mitochondrial damage further caused the release of cytochrome c and mitochondrial-related apoptosis proteins. Taken together, these results indicate that autophagy can help to clean up damaged mitochondria and play a protective role against $20(\mathrm{~S})$-GRh2-induced apoptosis. Inhibition of autophagy would aggravate mitochondrial damage, thus promoting mitochondriadependent apoptosis in ALL cells.
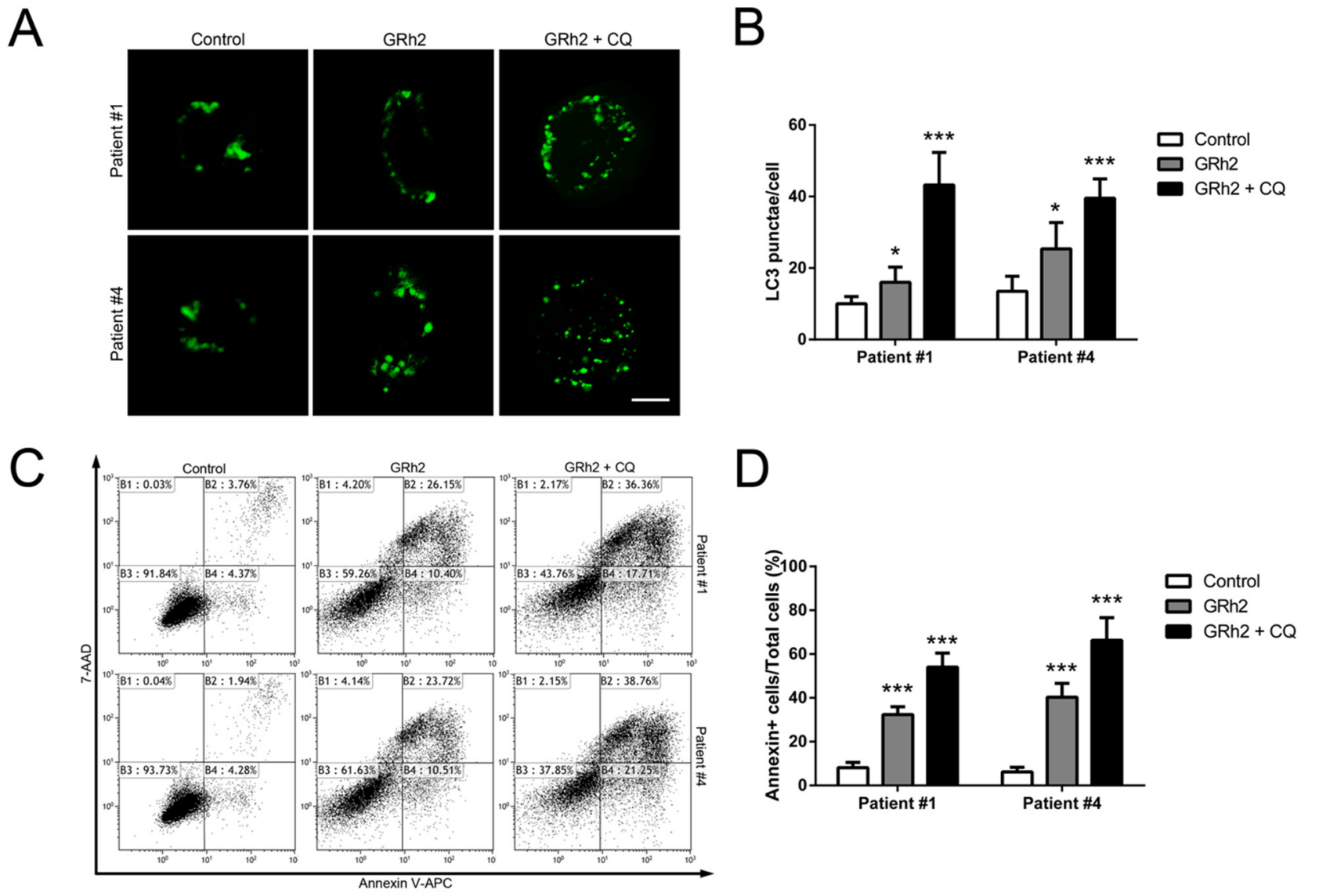

Figure 6: Autophagy plays a protective role in 20(S)-GRh2-induced apoptosis in human primary ALL cells. Cells were transfected with pEGFP-LC3 plasmids. After $24 \mathrm{~h}$ transfection, cells were treated with $40 \mu \mathrm{M} 20(\mathrm{~S})$-GRh2 in the presence or absence of CQ for 24 h. A. In primary B- ALL and T- ALL cells, the fluorescence of GFP-LC3 was examined by laser scanning confocal microscope $(\mathrm{Bar}=5 \mu \mathrm{M})$. B. GFP-LC3 puncta in each cell was determined and bar graphs are representative of 100 cells. Data are presented as mean \pm SEM $(\mathrm{n}=3)$ for each group. ${ }^{*} \mathrm{p}<0.05, * * \mathrm{p}<0.01$. C. The percentage of apoptotic cells was determined by FACS analysis. D. The percentage of Annexin V positive cells in every group was analyzed and graphed. Data are presented as mean \pm SEM ( $n=3$ ) for each group. $* \mathrm{p}<0.05, * * \mathrm{p}<0.01$. 
Moreover, we extended our study to find out whether autophagy played a role in 20(S)-GRh2induced apoptosis in primary ALL cells. We found that 20(S)-GRh2 treatment could increase autophagic flux in either primary B- ALL or T- ALL cells. Inhibition of autophagy by CQ sensitized those cancer cells towards 20(S)-GRh2. Therefore, our data suggest that inhibition of autophagy combined with 20(S)-GRh2 treatment would be more effective, and closer for future clinical application.

In view of the above arguments and the new data presented herein, we strongly propose that autophagy plays a protective role in 20 (S)-GRh2-induced apoptosis in ALL cell lines and human primary ALL cells. Either genetic or pharmacologic inhibition of autophagy can be more effective in reducing viability and enhancing 20(S)-GRh2-induced toxicity to ALL cells. In addition, inhibition of autophagy could accelerate mitochondriadependent apoptosis by stimulating mitochondrial ROS generation, promoting the loss of MMP and upregulation of mitochondria-dependent apoptosis in ALL cells. Collectively, this study suggests that inhibition of autophagy could potentiate anticancer property of 20(S)GRh2 and provide a novel strategy for ALL therapy in future.

\section{MATERIALS AND METHODS}

\section{Reagents and antibodies}

20(S)-GRh2 (purity 99.48\%) was purchased from Beijing North Carolina Chuanglian Biological Technology Research Istitute (Beijing, China). MitoTEMPO was obtained from Santa Cruz Biotechnology (Dallas, TX, USA), 3-MA, CQ and RAPA were purchased from Sigma.

The primary antibodies against rabbit cytochrome c, caspase-9, cleaved caspase-3, and the secondary horseradish peroxidase (HRP)-labeled goat-anti-mouse and goat-anti-rabbit antibodies, were purchased from Cell Signaling Technology (Danvers, MA, USA). Antibodies against rabbit cleaved caspase-9, GAPDH, $\beta$-actin, Tubulin were purchased from Bioworld Technology (NanJing, China). Antibodies against rabbit LC-3, Atg5 and beclin-1 were obtained from Abcam Company (Cambridge, MA, USA).

\section{Samples and cell culture}

Bone marrow (BM) samples were derived from 6 newly diagnosed cases, untreated patients with B-ALL and T-ALL. Peripheral blood (PB) samples were obtained from 3 healthy individuals. All human BM and PB samples were obtained with written informed consent. Mononuclear cells isolated from BM and PB through density gradient centrifugation on standard Ficoll-HyPaque. T-cells and B-cells were sorted from peripheral blood mononuclear cells (PBMC) by FACS (BD Bioscience Influx, Franklin Lakes, NJ, USA). In brief, the PBMCs were incubated with FITC-conjugated anti-CD3, PE-conjugated antiCD19 and purified by FACS to obtain the CD3 ${ }^{+}$T-cells and $\mathrm{CD} 19^{+} \mathrm{B}$ - cells, respectively. The purities of the sorted cells were $>95 \%$.

Human pre-B-ALL cell line Reh and T-ALL cell line Jacket were purchased from Cell Bank of Chinese Academic of Science (Shanghai, China). Cells were cultured at $37^{\circ} \mathrm{C}$ and 5\% CO2 in RPMI 1640 medium (Hyclone, Logan, Utah, USA), supplemented with $10 \%$ fetal bovine serum (FBS, Gibco, Grand Island, NY, USA), Penicillin/Streptomycin (1:100, Sigma, St. Louis, MO, USA).

\section{Cell viability assays}

Cell viability was determined by CCK-8 (Dojin Laboratories, Kumamoto, Japan) assay in different cells. Cells $\left(5 \times 10^{5} / \mathrm{mL}\right)$ were plated on a 96 -well $(100 \mu \mathrm{L} /$ well) microplate and treated with ligands for required time. Cells were added with $10 \mu \mathrm{L} \mathrm{CCK}-8$ solution, and incubated for $4 \mathrm{~h}$ at $37^{\circ} \mathrm{C}$. Absorbance in each well was quantified at $450 \mathrm{~nm}$ using an automated enzyme-linked immunosorbent assay (ELISA) reader (Tecan, Salzburg, Austria).

\section{The cytotoxicity assay}

Cells transfected with vector or ShATG5 plasmid were exposed to $40 \mu \mathrm{M}$ GRh2 for $24 \mathrm{~h}$ and stained

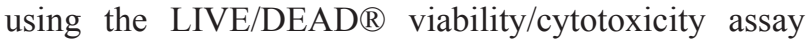
kit (Invitrogen, Carlsbad, CA, USA) to analyze for the presence of live or dead cells. Briefly, calcein AM probe is conversed to the intensely fluorescent calcein in living cells, producing a green fluorescence. While, EthD-1 probe enters cells with damaged membranes, producing a red fluorescence by binding to nucleic acids. Cells were then stained with nuclear Hoechst 33342. The percentage of dead cells with EthD-1 probe was determined in highpower fields of each sample. Images were analyzed using Image J. A minimum 500 cells were counted.

\section{Annexin V/7-amino-actinomycin D (7-AAD) flow cytometry assay}

Cells were treated with different ligands for required time. Cells were washed twice with cold PBS and resuspended in binding buffer $500 \mu \mathrm{L}$. Annexin V-APC and 7-Amino-actinomycin D (7-AAD, BD Pharmingen, San Diego, CA, USA) were added, away from light for $15 \mathrm{~min}$ at room temperature. The cells were immediately evaluated by flow cytometry (FACScan; Becton Dickinson, San Diego, CA, USA) within one hour. Annexin $\mathrm{V}+$ and 7-AAD- cells were designated as early apoptotic cells and Annexin $\mathrm{V}+$ and 7-AAD + cells were designated as late apoptotic cells. 


\section{Detection of autophagic vacuoles by MDC staining}

A fluorescent compound, MDC (Sigma, St. Louis, MO, USA), has served as a useful fluorescent marker for autophagic vacuoles. Reh cells $\left(5 \times 10^{5} /\right.$ well $)$ were cultured in 6-well culture plates and treated without or with 20(S)GRh2 $(20$ and $40 \mu \mathrm{M})$ for $24 \mathrm{~h}$, then incubated with 50 $\mu \mathrm{M}$ MDC in PBS at $37^{\circ} \mathrm{C}$ for $1 \mathrm{~h}$. After washing with PBS, the stained cells were immediately observed under a laser scanning confocal microscope (Zeiss, Germany) and analyzed by flow cytometry.

\section{Detection of GFP-LC3}

Human pEGFP-LC3 plasmid (Addgene plasmid 24920), which encodes a fusion protein of enhanced green fluorescent protein (EGFP) and LC3, was obtained from Addgene (Cambridge, MA, USA). Cells were seeded in 12-well plates and transfected with pEGFP-LC3 plasmid using Lipofectamine 2000 (Invitrogen) following the manufacturer's instructions. After $24 \mathrm{~h}$ transfection, cells were treated with different ligands for $24 \mathrm{~h}$. The fluorescence of GFP-LC3 was examined by laser scanning confocal microscope. Autophagic flux was determined by evaluating the punctuated pattern of GFP (punctae/cell were counted).

\section{Knockdown of ATG5}

ATG5 expression was knockdown by transfected with shRNA retroviruses. The shRNA and sequence was 5'-GCCATCAATCGGAAACTCATG-3'. Retrovirus particles were obtained in HEK293T cells according to the manufacturer's instructions. Cells were seeded into sixwell plates and transfected with either empty or shRNA retroviruses using X-tremeGENE HP reagent (Roche Applied Science, Indianapolis, IN, USA), then stably transduced cells were selected by puromycin antibiotics in the medium.

\section{Measurement of mitochondrial ROS generation}

The formation of mitochondrial ROS was measured using MitoSOX ${ }^{\mathrm{TM}}$ Red (Life technologies) assay. MitoSOX ${ }^{\mathrm{TM}}$ Red reagent is oxidized by superoxide once inside the mitochondria, and is converted to a fluorogenic oxidation product upon binding to nucleic acids [39]. For microscopy, cells were treated with $40 \mu \mathrm{M}$ GRh2 for $24 \mathrm{~h}$ in the presence or absence of $50 \mu \mathrm{M}$ mitoTEMPO. After staining with MitoSOX ${ }^{\mathrm{TM}}$ Red, cells were stained with Hoechst 33342. Microscopic images of fluorescence were collected using a fuorescence microscopy (IX-81; Olympus Corp., Tokyo, Japan). For flow cytometry, cells were treated with different ligands for $24 \mathrm{~h}$. After staining, MitoSOX ${ }^{\mathrm{TM}}$ Red fluorescence was analyzed by flow cytometry.

\section{Detection of mitochondrial membrane potential}

Cells were cultured in 12 -well plates $\left(5 \times 10^{5} /\right.$ well) and treated with different ligands. In ALL cells, fluorescent probe JC-1 (Sigma, Louis, MO, USA) was used to estimate MMP. In cells treated with vector or shATG5, the MMP was determined using the fluorescent MitoTracker probe (Invitrogen). After $24 \mathrm{~h}$ incubation, cells were stained with JC-1 or MitoTracker in the dark at $37^{\circ} \mathrm{C}$ for $30 \mathrm{~min}$ and immediately observed under a fuorescence microscopy or analyzed by a FACScan flow cytometer.

\section{Transmission electron microscopy}

After treatment with $40 \mu \mathrm{M} \mathrm{GRh} 2$ for $24 \mathrm{~h}$, Reh and Jurkat cells, including control, were collected and fixed with $2.5 \%$ glutaraldehyde solution ( $\mathrm{pH} 7.4$ ) for $2 \mathrm{~h}$, postfixed with $1 \%$ osmium tetroxide for $1 \mathrm{~h}$, and then washed. After dehydration in a growing gradient of ethanol $(30,50$, 70 and $95 \%, 5$ min for each step), impregnation steps and inclusion were performed in Epon and finally polymerized at $60^{\circ} \mathrm{C}$ for $48 \mathrm{~h}$. Ultrathin sections were stained with uranyl acetate and lead citrate for observation under a Tecnai G2 Spirit Twin transmission electron microscope (FEI, USA) at an accelerating voltage of $80 \mathrm{kV}$.

\section{Western blot analysis}

After treatment, cells were collected and lysed for $30 \mathrm{~min}$ in RIPA buffer. The samples were heated to $100^{\circ} \mathrm{C}$ for $5 \mathrm{~min}$ and placed briefy on ice. A total of 20 $\mu \mathrm{L}$ of the supernatant was loaded onto a $12 \%$ sodium dodecyl sulfate-polyacrylamide gel electrophoresis (SDS-PAGE) gel. After electrophoresis, the protein was electrotransferred to a Hybond-ECL polyvi-nylidene fuoride membrane. The membrane was blocked with $5 \%$ milk for $1 \mathrm{~h}$, and incubated overnight with primary antibodies, followed by horseradish peroxidase (HRP)conjugated secondary antibodies for $1 \mathrm{~h}$ at room temperature. The membranes were incubated with HRP-conjugated secondary antibodies for $1 \mathrm{~h}$ at room temperature. The immunoreactive bands were detected with an enhanced chemiluminescence.

\section{Statistical analyses}

All data were expressed as the means \pm the standard error of the mean (SEM). Statistical analyses were performed using Prism 5.03 (GraphPad Software Inc., San Diego, CA, U.S.A.). The data were examined using Student's t-test or one-way ANOVA followed by the Bonferroni post hoc test. A p value less than 0.05 was considered significant. The levels of significance were set at $\mathrm{p}<0.05, \mathrm{p}<0.01$ or $\mathrm{p}<0.001$. 


\section{ACKNOWLEDGMENTS}

This work was supported by the National Natural Science Foundation of China (No. 81570140, No. 21307039), National High Technology Research and Development Program of China (2013AA102106), Program for Changjiang Scholars and Innovative Research Team in University (IRT15R49). We thank Andy Peng Xiang from the Center for Stem Cell Biology and Tissue Engineering for their excellent technical assistance.

\section{CONFLICTS OF INTEREST}

The authors declare no conflicts of interest.

\section{REFERENCES}

1. Lo Nigro L. Biology of childhood acute lymphoblastic leukemia. J Pediatr Hematol Oncol. 2013; 35:245-52.

2. Pui $\mathrm{CH}$, Robison LL, Look AT. Acute lymphoblastic leukaemia. Lancet. 2008; 371: 1030-1043.

3. Richardson RB. Promotional etiology for common childhood acute lymphoblastic leukemia: The infective lymphoid recovery hypothesis. Leuk Res. 2011; 35:1425-1431.

4. Inaba $\mathrm{H}$, Greaves $\mathrm{M}$, Mullighan $\mathrm{CG}$. Acute lymphoblastic leukaemia. Lancet. 2013; 381:1943-1955.

5. Barisone GA, Satake N, Lewis C, Duong C, Chen C, Lam KS, Nolta J, Díaz E. Loss of MXD3 induces apoptosis of Reh human precursor B acute lymphoblastic leukemia cells. Blood Cells Mol Dis. 2015; 54:329-335.

6. Robison LL. Late effects of acute lymphoblastic leukemia therapy in patients diagnosed at 0-20 years of age. Hematology Am Soc Hematol Educ Program. 2011; 2011:238-242.

7. Stocco G, Franca R, Verzegnassi F, Londero M, Rabusin M, Decorti G. Pharmacogenomic approaches for tailored anti-leukemic therapy in children. Curr Med Chem. 2013; 20:2237-2253.

8. Wang S, Wu X, Tan M, Gong J, Tan W, Bian B, Chen M, Wang Y. Fighting fire with fire: poisonous Chinese herbal medicine for cancer therapy. J Ethnopharmacol. 2012;140:33-45.

9. Kim DG, Jung KH, Lee DG, Yoon JH, Choi KS, Kwon SW, Shen HM, Morgan MJ, Hong SS, Kim YS. 20(S)-Ginsenoside Rg3 is a novel inhibitor of autophagy and sensitizes hepatocellular carcinoma to doxorubicin. Oncotarget. 2014; 5:4438-4451. doi: 10.18632/oncotarget.2034.

10. Wang Y, You J, Yu Y, Qu C, Zhang H, Ding L, Zhang H, Li X. Analysis of ginsenosides in Panax ginseng in high pressure microwave-assisted extraction. Food Chem. 2008; 110:161-167.
11. Lü JM, Yao Q, Chen C. Ginseng compounds: an update on their molecular mechanisms and medical applications. Curr Vasc Pharmacol. 2009; 7:293-302.

12. Kang KS, Ham J, Kim YJ, Park JH, Cho EJ, Yamabe N. Heat-processed Panax ginseng and diabetic renal damage: active components and action mechanism. J Ginseng Res. 2013; 37:379-388.

13. Popovich DG, Kitts DD. Structure-function relationship exists for ginsenosides in reducing cell proliferation and inducing apoptosis in the human leukemia (THP-1) cell line. Archives of Biochemistry and Biophysics. 2002; 406:1-8.

14. Guo XX, Guo Q, Li Y, Lee SK, Wei XN, Jin YH. Ginsenoside Rh2 induces human hepatoma cell apoptosisvia bax/bak triggered cytochrome C release and caspase-9/ caspase-8 activation. Int J Mol Sci. 2012; 13:15523-15535.

15. Choi S, Oh JY, Kim SJ. Ginsenoside Rh2 induces Bcl-2 family proteins-mediated apoptosis in vitro and in xenografts in vivo models. J Cell Biochem. 2011; 112:330-340.

16. Tang XP, Tang GD, Fang CY, Liang ZH, Zhang LY. Effects of ginsenoside $\mathrm{Rh} 2$ on growth and migration of pancreatic cancer cells. World J Gastroenterol. 2013; 19: 1582-1592.

17. Zhou B, Xiao X, Xu L, Zhu L, Tan L, Tang H, Zhang Y, Xie Q, Yao S. A dynamic study on reversal of multidrug resistance by ginsenoside $\mathrm{Rh} 2$ in adriamycin-resistant human breast cancer MCF-7 cells. Talanta. 2012; 88:345-351.

18. Liu J, Shimizu K, Yu H, Zhang C, Jin F, Kondo R. Stereospecificity of hydroxyl group at C-20 in antiproliferative action of ginsenoside $\mathrm{Rh} 2$ on prostate cancer cells. Fitoterapia. 2010; 81:902-905.

19. Nakata H, Kikuchi Y, Tode T, Hirata J, Kita T, Ishii K, Kudoh K, Nagata I, Shinomiya N. Inhibitory effects of ginsenoside $\mathrm{Rh} 2$ on tumor growth in nude mice bearing human ovarian cancer cells. Jpn J Cancer Res. 1998; 89:733-740.

20. Zhu Y, Xu J, Li Z, Xie S, Zhou J, Guo X, Zhou X, Li G, Zhong $\mathrm{R}$, Ma A. Ginsenoside Rh2 suppresses growth of uterine leiomyoma in vitro and in vivo and may regulate $\mathrm{ER} \alpha / \mathrm{c}-\mathrm{Src} /$ p38 MAPK activity. J Funct Foods. 2015; 18: 73-82.

21. Cheong JH, Kim H, Hong MJ, Yang MH, Kim JW, Yoo H, Yang H, Park JH, Sung SH, Kim HP, Kim J. Stereoisomerspecific anticancer activities of ginsenoside Rg3 and Rh2 in HepG2 Cells: disparity in cytotoxicity and autophagyinducing effects due to 20(S)-epimers. Biol Pharm Bull. 2015; 38:102-108.

22. Xia T, Wang JC, Xu W, Xu LH, Lao CH, Ye QX, Fang JP. 20S-Ginsenoside Rh2 induces apoptosis in human Leukaemia Reh cells through mitochondrial signaling pathways. Biol Pharm Bull. 2013; 37:248-254. 
23. Hanahan D, Weinberg RA. Hallmarks of cancer: the next generation. Cell. 2011; 144:646-674.

24. Hengartner MO. The biochemistry of apoptosis. Nature. 2000; 407:770-776.

25. Ouyang L, Shi Z, Zhao S, Wang FT, Zhou TT, Liu B, Bao JK. Programmed cell death pathways in cancer: a review of apoptosis, autophagy and programmed necrosis. Cell Prolif. 2012; 45:487-98.

26. Green DR, Kroemer G. The pathophysiology of mitochondrial cell death. Science. 2004; 305:626-629.

27. Green DR, Kroemer G. Pharmacological manipulation of cell death: clinical applications in sight? J Clin Invest. 2005; 115:2610-2617.

28. Kroemer G, Galluzzi L, Brenner C. Mitochondrial membrane permeabilization in cell death. Physiol Rev. 2007; 87: 99-163.

29. Simon HU, Haj-Yehia A, Levi-Schaffer F. Role of reactive oxygen species (ROS) in apoptosis induction. Apoptosis. 2000; 5:415-418.

30. Heinicke U, Kupka J, Fulda S. JNJ-26481585 primes rhabdomyosarcoma cells for chemotherapeutics by engaging the mitochondrial pathway of apoptosis. Oncotarget. 2015; 6:37836-37851. doi: 10.18632/oncotarget.6097.

31. Karna P, Zughaier S, Pannu V, Simmons R, Narayan S, Aneja R. Induction of reactive oxygen species-mediated autophagy by a novel microtubule-modulating agent. J Biol Chem. 2010;285:18737-18748.

32. Yang YP, Liang ZQ, Gu ZL, Qin ZH. Molecular mechanism and regulation of autophagy. Acta Pharmacol Sin.2005; 26:1421-1434.

33. Pyo JO, Nah J, Jung YK. Molecules and their functions in autophagy. Exp Mol Med. 2012; 44:73-80.

34. Jain MV, Paczulla AM, Klonisch T, Dimgba FN, Rao SB, Roberg K, Schweizer F, Lengerke C, Davoodpour P, Palicharla VR, Maddika S, Łos M. Interconnections between apoptotic, autophagic and necrotic pathways: implications for cancer therapy development. J Cell Mol Med. 2013; 17:12-29.

35. White E. Deconvoluting the context-dependent role for autophagy in cancer. Nat Rev Cancer. 2012; 12:401-410.

36. Bredholt $\mathrm{T}$, Dimba EA, Hagland HR, Wergeland L, Skavland J, Fossan KO, Tronstad KJ, Johannessen AC, Vintermyr OK, Gjertsen BT. Camptothecin and khat (Catha edulisForsk.) induced distinct cell death phenotypes involving modulation of c-FLIPL, Mcl-1, procaspase- 8 and mitochondrial function in acute myeloid leukemia cell lines. Mol Cancer. 2009; 8:101.

37. Huang HL, Chen YC, Huang YC, Yang KC, Pan Hy, Shih SP, Chen YJ. Lapatinib induces autophagy, apoptosis and megakaryocytic differentiation in chronic myelogenous leukemia K562 cells. PLoS One. 2011; 6:e29014.

38. Pan T, Rawal P, Wu Y, Xie W, Jankovic J, Le W. Rapamycin protects against rotenone-induced apoptosis through autophagy induction. Neuroscience. 2009; 164:541-551.
39. Pui CH, Mullighan CG, Evans WE, Relling MV. Pediatric acute lymphoblastic leukemia: where are we going and how do we get there? Blood. 2012; 120:1165-1174.

40. Wang W, Zhao Y, Rayburn ER, Hill DL, Wang H, Zhang R. In vitro anti-cancer activity and structure-activity relationships of natural products isolated from fruits of Panax ginseng. Cancer Chemother Pharmacol. 2007; 59:589-601.

41. Voruganti S, Qin JJ, Sarkar S, Nag S, Walbi IA, Wang S, Zhao Y, Wang W, Zhang R. Oral nano-delivery of anticancer ginsenoside 25-OCH3-PPD, a natural inhibitor of the MDM2 oncogene: Nanoparticle preparation, characterization, in vitro and in vivo anti-prostate cancer activity, and mechanisms of action. Oncotarget. 2015; 6:21379-21394. doi: 10.18632/oncotarget.4091.

42. Kim MJ, Yun H, Kim DH, Kang I, Choe W, Kim SS, Ha J. AMP-activated protein kinase determines apoptotic sensitivity of cancer cells to ginsenoside-Rh2. J Ginseng Res. 2014; 38:16-21.

43. Li S, Guo W, Gao Y, Liu Y. Ginsenoside Rh2 inhibits growth of glioblastoma multiforme through mTor. Tumour Biol. 2015; 36:2607-2612.

44. Park HM, Kim SJ, Kim JS, Kang HS. Reactive oxygen species mediated ginsenoside $\mathrm{Rg} 3$ - and Rh2-induced apoptosis in hepatoma cells through mitochondrial signaling pathways. Food Chem Toxicol. 2012; 50:2736-2741.

45. Li B, Zhao J, Wang CZ, Searle J, He TC, Yuan CS, Du W. Ginsenoside Rh2 induces apoptosis and paraptosis-like cell death in colorectal cancer cells through activation of p53. Cancer Lett. 2011; 301: 185-192.

46. Radogna F, Dicato M, Diederich M. Cancer-type-specific crosstalk between autophagy, necroptosis and apoptosis as a pharmacological target. Biochem Pharmacol. 2015; 94:1-11.

47. Tsai JP, Lee CH, Ying TH, Lin CL, Lin CL, Hsueh JT, Hsieh YH. Licochalcone A induces autophagy through $\mathrm{PI} 3 \mathrm{~K} / \mathrm{Akt} / \mathrm{mTOR}$ inactivation and autophagy suppression enhances Licochalcone A-induced apoptosis of human cervical cancer cells. Oncotarget. 2015; 6:28851-28866. doi: 10.18632/oncotarget.4767.

48. Maiuri MC, Zalckvar E, Kimchi A, Kroemer G. Self-eating and self-killing:crosstalk between autophagy and apoptosis. Nat Rev Mol Cell Biol. 2007; 8:741-752.

49. Ouyang C, You J, Xie Z. The interplay between autophagy and apoptosis in the diabetic heart. J Mol Cell Cardiol. 2014; 71:71-80.

50. Mizushima N, Levine B, Cuervo AM, Klionsky DJ. Autophagy flights disease through cellular self-digestion. Nature. 2008; 451:1069-1075.

51. Bincoletto C, Bechara A, Pereira GJ, Santos CP, Antunes F, Peixoto da-Silva J, Muler M, Gigli RD, Monteforte PT, Hirata H, Jurkiewicz A, Smaili SS. Interplay between apoptosis and autophagy, a challenging puzzle: New perspectives on antitumor chemotherapies. Chem Biol Interact. 2013; 206: 279-288. 
52. Zhang Y, Wu Y, Tashiro S, Onodera S, Ikejima T. Involvement of PKC signal pathways in oridonin-induced autophagy in HeLa cells: A protective mechanism against apoptosis. Biochem Biophys Res Commun. 2009; 378:273-278.

53. Jin CY, Yu HY, Park C, Han MH, Hong SH, Kim KS, Lee YC, Chang YC, Cheong J, Moon SK, Kim GY, Moon HI, Kim WJ,et al. Oleifolioside B-mediated autophagy promotes apoptosis in A549 human non-small cell lung cancer cells. Int J Oncol. 2013; 43:1943-1950.

54. Ashrafi G, Schwarz TL. The pathways of mitophagy for quality control and clearance of mitochondria. Cell Death Differ. 2013; 20:31-42.
55. Suski JM, Lebiedzinska M, Bonora M, Pinton P, Duszynski J, Wieckowski MR. Relation between mitochondrial membrane potential and ROS formation. Methods Mol Biol. 2012; 810:183-205.

56. Rikiishi H. Novel Insights into the Interplay between Apoptosis and Autophagy. Int J Cell Biol. 2012; 2012:317645.

57. Wang K, Klionsky DJ. Mitochondria removal by autophagy. Autophagy. 2011; 7:297-300.

58. Eisenberg-Lerner A, Bialik S, Simon HU, Kimchi A. Life and death partners: apoptosis, autophagy and the cross-talk between them. Cell Death Differ. 2009; 16:966-975. 